Int. J. Morphol.,

31(2):672-680, 2013.

\title{
Dimensión Vertical en Edentados: Relación con Síntomas Referidos
}

\author{
Vertical Dimension on Edentulous Patient: Relationship with Symptoms Reported
}

Ramirez, L. M. , Echeverría, P.**; Zea, F. J.**** \& Ballesteros, L. E. ${ }^{* * * * *}$

\begin{abstract}
RAMÍREZ, L. M.; ECHEVERRÍA, P.; ZEA, F. J. \& BALLESTEROS, L. E. Dimensión vertical en edentados: relación con síntomas referidos. Int. J. Morphol., 31(2):672-680, 2013.

RESUMEN: La dimensión vertical es una medida aproximada de las relaciones fisiológicas intermaxilares. Su obtención es un proceso sensible y metódico que produce efectos craneofaciales colaterales cuando es erróneamente lograda. Los conceptos individualistas de su obtención deben ser cambiados e interiorizados de una manera colectiva debido a la complejidad de la dinámica masticatoria que exige una perspectiva más amplia de esta noción. La correspondencia entre la dimensión vertical y lo funcional-disfuncional cráneocervical es innegable y va mas allá de una relación exclusivamente dental. Esta revisión busca situar al lector en una realidad estomatognática y no solo odontológica, que exige mayores esfuerzos y un cambio de perspectiva en la conceptualización de la importancia de la dimensión vertical en el normal funcionamiento del sistema estomatognático y de estructuras vecinas en el paciente edentado.
\end{abstract}

PALABRAS CLAVE: Dimensión vertical; Síntomas referidos; Tinitus; Vértigo; Otalgia; Cefalea; Hipoacusia.

\section{INTRODUCCIÓN}

No poder morder los alimentos con firmeza y no reír o hablar despreocupadamente para los usuarios de prótesis total, bien puede considerarse en las primeras diez situaciones que incomodan a la mayoría de la población adulta mayor (algunas comorbidas entre ellas) y que ajustadamente entrarían en el marco de los problemas prioritarios en salud pública.

Lo que la mayoría de las personas con prótesis total espera de ellas, es poder morder sin dolor, cuando es apenas lógico que así sea. Una prótesis total bien construida no solo debe estar exenta de producirlo, sino también ofrecer una excelente estética con retención, soporte y estabilidad sobre los tejidos que la toleran, ser una restauración con volúmenes y dimensiones craneales que recupere las pérdidas ocasionadas por el tiempo. Esta última consideración que atañe principalmente a la dimensión vertical (DV) o altura facial, se ha convertido en un reto de difícil conquista en los profesionales de nuestra disciplina. El concepto de DV parece ser un constructo académico de difícil recordación y ponderación clínica. Esto se ve evidenciado en pacientes que probablemente han recibido durante su vida varios juegos de prótesis mal fabricadas con fallas justamente en la restitución de esta. Curiosamente, estos pacientes con características faciales dramáticamente pérdidas en el tiempo y con prótesis mal diseñadas (Fig. 1), parecieran haber olvidado las proporciones juveniles de su rostro y apaciblemente aceptan la reflexión final de su odontólogo que seguramente procede sin premeditación pero con apremio.

Para un paciente, recapacitar sobre las proporciones faciales originarias juveniles y recordar el volumen de sus labios, la forma de su perfil o la línea de sonrisa y surcos naturales de su otrora dentado rostro; así como la forma y el tamaño único de sus dientes, es un ejercicio de mucha complejidad sino imposible. En la práctica clínica parece que lo único a tener en cuenta por ellos es que su prótesis sea modestamente bonita, fácil y rápida de "amansar" sin mucho dolor. Por supuesto, la confianza al morder, la articulación de los sonidos en la pronunciación de los fonemas para hablar con seguridad o la eliminación de síntomas referidos

\footnotetext{
* Odontólogo, Rehabilitador Oral, Magister en Ciencias Básicas Biomédicas. Grupo Estomatología Biomédica. Profesor Asociado Facultad de Odontologia. Universidad de Antioquia, Medellín, Colombia.

** Odontóloga Prostodoncista. Profesor Titular Facultad de Odontologia. Universidad de Antioquia, Medellín, Colombia.

*** Odontólogo Integral del Adulto. Profesor Facultad de Odontologia. Universidad de Antioquia, Medellín, Colombia.

***** Medico, Magister en Ciencias Básicas Biomédicas. Profesor Titular Facultad de Salud. Universidad Industrial de Santander, Bucaramanga, Colombia.
} 
craneofaciales por esta problemática, son igualmente significativos, pero no ponderado por los pacientes. Todas estas consideraciones asociadas a la DV, deben ser entendidas y traducidas por el odontólogo en sus pacientes. Una visión con criterio clínico restaurador y morfofisiológico, más que uno mecanicista protésico, debe permitir profundizar en todos los referentes asociados a la construcción y rehabilitación de un paciente edentado y con miras a suplir más allá de lo morfológico dental individual, lo estético y funcional en el marco de una visión dinámica y compuesta craneofacial y dental. La satisfacción estará entonces asociada a la comodidad y de seguro podrá ser medida en una escala que represente la adherencia al tratamiento de los pacientes con prótesis de remplazo.

En esta revisión se busca matizar la importancia de la DV en la construcción de prótesis total. Se hace énfasis en la capacidad dañina que tiene la mala restauración de la DV en la etiología de síntomas cráneo-cervicales y óticos referidos, igualmente la fisiopatología asociada al desarrollo de los síntomas secundarios referidos o proyectados (heterotópicos) que explican la mecánica y procesos físicos asociados a esta sintomatología a partir de una prótesis total erróneamente construida. Finalmente, se muestra una técnica simple y precisa que facilita su obtención final de forma sistemática, progresiva y controlada.

\section{Rehabilitación de la DV en un adulto mayor edentado.} El grado de dificultad de este procedimiento se hace evidente al considerar que la prótesis total se aloja en maxilares mucosos rodeados de estructuras osteo-artro-musculares que se mueven permanentemente ante la demanda masticatoria. Estos maxilares sin puntos de referencia fijos o estables, como los dientes, representan un reto de cuidado por restaurar. Consecuentemente, esta cualidad móvil de los maxilares hace fina la tarea de reconstruir formas y alturas originales; y si a lo anterior se suma que el tiempo ha dejado huella de desgaste en los rebordes maxilares, la tarea

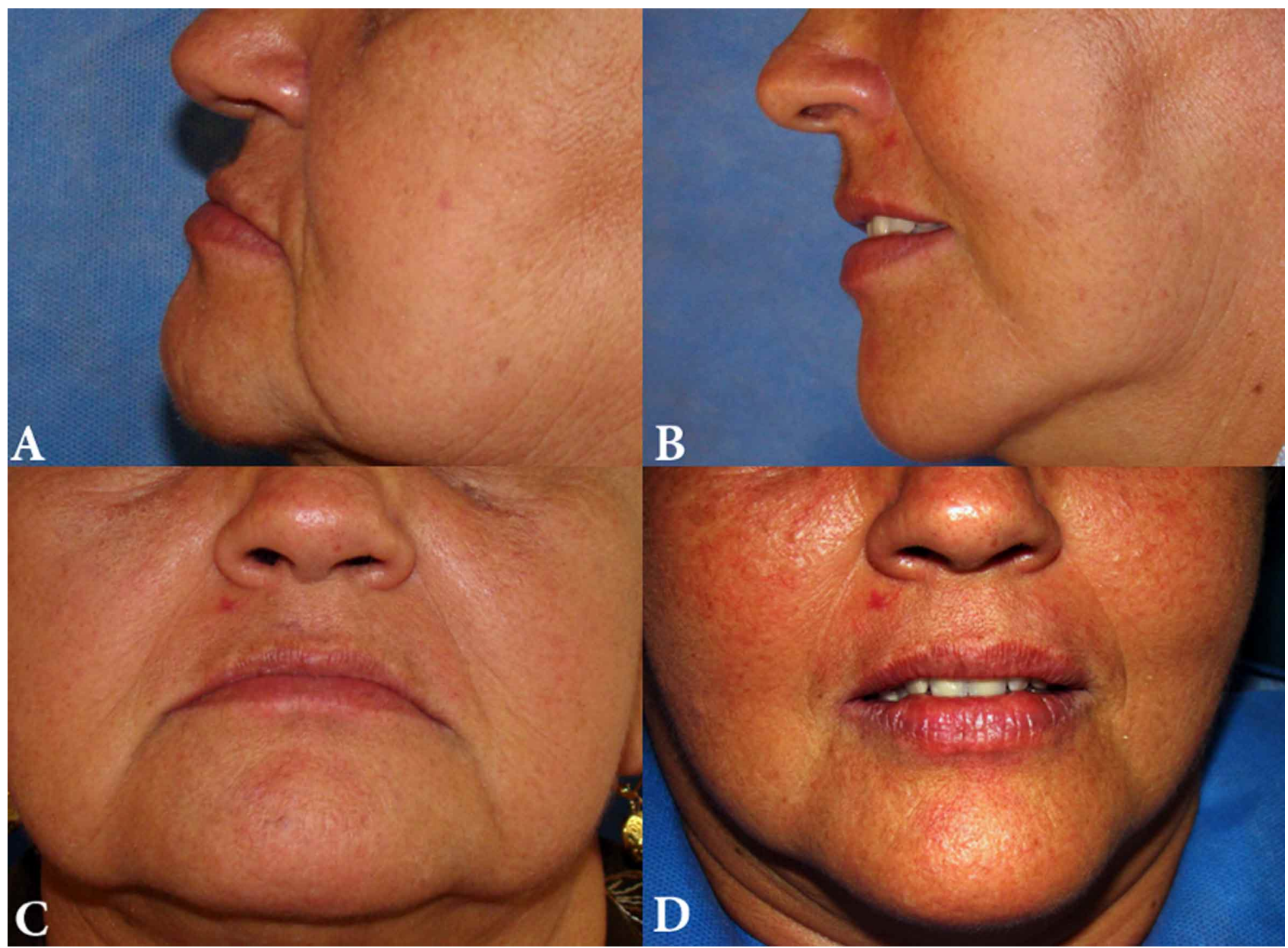

Fig. 1. Registro fotográfico lateral y frontal de paciente con prótesis total bimaxilar programada con dimensión vertical incorrecta (A, C) y la misma paciente después de restaurada apropiadamente (B, D). Fotos de consulta privada y permitidas por la paciente. 
restaurativa se vuelve colosal; es así entonces que la técnica, la comprensión morfofisiológica del sistema masticatorio, el laboratorio y la práctica son imperantes en la elaboración de prótesis adecuadas. Lo que se busca al reconstruir la DV pérdida es justamente recuperar las proporciones faciales alteradas (Fig. 2): mentón próximo a la nariz y hacia adelante, el soporte y márgenes de los labios perdidos, una ubicación de las comisuras labiales curvadas inferiormente y lejos del origen muscular, la falta de tonicidad muscular y una cara flácida consecuente, con pliegues pronunciados y comisuras sin volumen, todo esto sumado a los efectos colaterales originados en la pérdida de la DV dan cuenta del colapso facial evidente en estos pacientes.

La calidad de los materiales y la experiencia del laboratorio indiscutiblemente son trascendentes; sin embargo, la comunicación entre operador y laboratorio es más importante; el diálogo y asesoría permanente que evite resultados no programados debe ser la regla de oro. Desafortunadamente, lo que sucede en la actualidad, en la práctica clínica, es que las decisiones más trascendentes en la elaboración de una prótesis total terminan endosadas al técnico del laboratorio dental, cuando cada paso de esta fabricación merece vigilancia y control del operador y colaboración del técnico. Cuando se construye una prótesis total conectada a implantes (sobredentaduras o prótesis híbrida), éste dialogo se hace más crítico e imperativo, similar al diálogo entre rehabilitador y cirujano. Las características y exigencias individuales de cada paciente, estudiadas, planeadas y programadas, es el derrotero y guía en ambas situaciones.

Consideraciones biológicas de las relaciones verticales de los maxilares. Se debe comenzar por aceptar que no hay una DV exacta y que ésta se determina fisiológicamente por la longitud de las fibras de los músculos agonistas de la apertura y del cierre mandibular (conforme a la longitud dental), lo que en un principio representa un grado de dificultad con- siderable para la rehabilitación de un paciente con prótesis total. Los músculos, mucosas y articulaciones constantemente envían información de mecanocepción y propiocepción hacia los centros reguladores centrales que permiten lograr una posición promedio y variable a través de mecanismos automáticos miotáticos basados en la generación de patrones musculares centrales y que se revierten en una DV constantemente ajustada a las demandas del entorno físico, espacial y temporal. Una serie de impulsos excitadores e inhibidores desde diferentes niveles segmentarios centrales regulan la dimensión vertical modulada en orígenes extrapiramidales y piramidales corticales, cerebelosos, reticulares, y varios periféricos (dento-muco-osteo-artro-musculares), sin dejar de lado a los ganglios basales. Por otro lado, los músculos de la masticación no se encuentran aislados de una dinámica cráneo-cervical compleja que tiene de manera recíproca y concomitante efectos sobre la postura y relaciones de horizontalidad y de verticalidad entre cabeza y cuello pero de forma similar entre mandíbula y maxilares.

La complejidad en lo anterior no es óbice para el logro de una DV correcta, por lo tanto, esta no debe ser el resultado del criterio personal apresurado del operador $\mathrm{y}$, antes por el contrario, deber ser lograda a través del análisis de diferentes posiciones ensayadas con antelación y calibradas con tiempo, que busquen establecer una correcta rehabilitación. Normalmente, en prótesis total se busca conseguir un equilibrio entre estética, función y comodidad sin ponderar a la DV como vital protagonista de estas tres dimensiones. Boucher (1997) señala que el uso de un tiempo apropiado destinado al ensayo de varias prótesis terapéuticas que incrementen la DV poco a poco, permitiría acostumbrar al paciente a cambios progresivos y ordenados en la recuperación paulatina de los rangos de la DV, sin embargo, esto puede aumentar considerablemente el costo y tiempo de elaboración de los trabajos protésicos; misma razón por la que la prerrogativa de esta en la practica clínica comúnmente se vuelve una quimera.

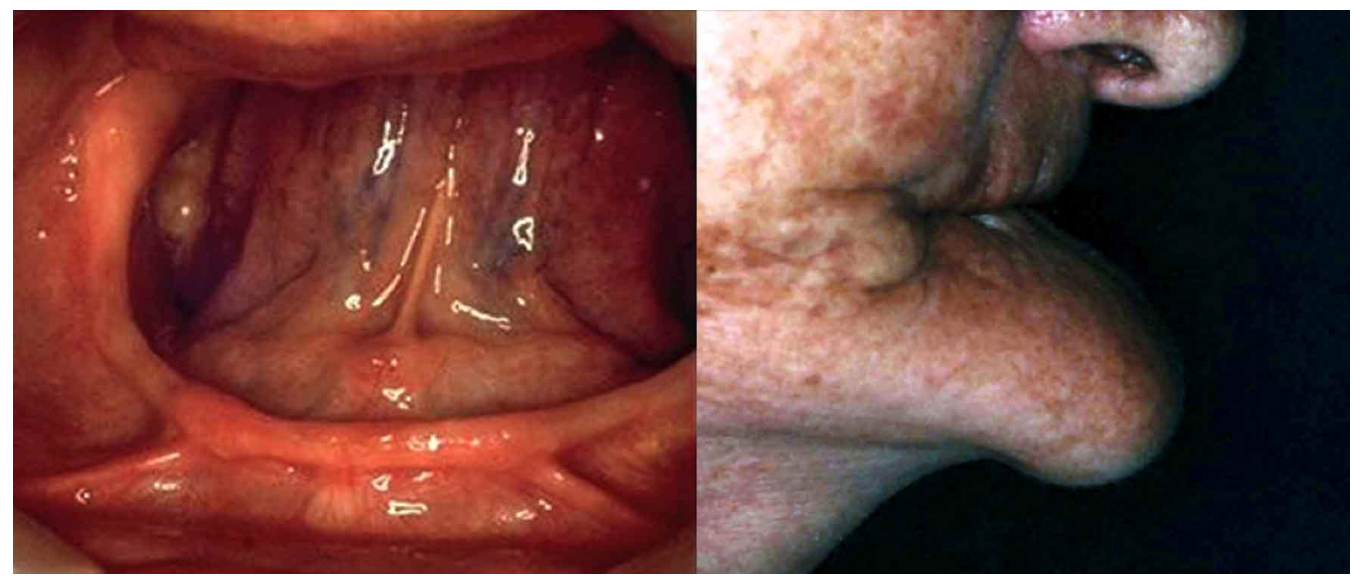

Fig. 2. Vista intraoral y extraoral de pérdida de la relación de la DV en un paciente edéntado. Fotos de consulta privada y permitidas por la paciente. 
Es fundamental advertir entonces que la DV es una distancia fisiológica entre mandíbula y maxilar que abarca un rango de espacio postural de descanso y fonación inoclusiva y que no es una posición estable y única que se puede lograr en un margen de minutos. Esta es modificada por la actividad dinámica del ambiente perioral y gravitacional, e igualmente por la naturaleza visco-elástica tisular, el peso mandibular, la presión negativa oral (Espacio de Donders), la postura corporal, la fatiga muscular y la dimensión cognitiva-emocional; todo en el marco de una retroalimentación sensorial y central periférica. Estas condicionantes la cualifican como relativa en un rango de medida y no absoluta como una medida exacta, lo que representa una gran fuente de error en su registro. En tal sentido, la influencia de la columna cervical y sus músculos en el sistema masticatorio son prácticamente ignorados y se les atribuye solo una función postural, cuando realmente cumplen una función importante en la posición dental entre maxilar y mandíbula asociada a la DV. La posición mandibular es influenciada por la postura del cuerpo en el espacio y viceversa (Makofsky, 1989). La posición de la cabeza es considerablemente dinámica con efectos sobre la DV y sobre la posición de contacto muscular y dental. Evidentemente, los cambios en la postura cervical pueden afectar la trayectoria de cierre mandibular, la posición postural o de descanso mandibular y la actividad muscular masticatoria, todas estas influenciando la DV.

Si se logra una DV sostenida por una posición mandibular óptima que mantenga los cambios posicionales del maxilar en sentido vertical y horizontal y que active mecanoreceptores periodontales, mucosos y reflejos propioceptivos musculares y articulares; la columna vertebral en toda su extensión responde, sufriendo cambios que son correspondientes a los obtenidos entre maxilar y mandíbula (Manns et al., 1979). Interesantemente, también se ha encontrado como la potencia muscular de miembros superiores, tronco y miembros inferiores se modifican en proporción directa a cambios en la DV e igualmente como la dorsi-planti flexión y posición de los pies puede ser modificada a través de cambios en ésta (Yoshino et al., 2003).

La DV perdida por edentulismo, iatrogenia o por desgaste dental avanzado, puede perturbar el equilibrio dinámico del sistema estomatognático ya que el componente de estabilidad músculo-articular que brindan los dientes puede verse afectado por la ausencia de éstos. La alteración de la DV igualmente puede producir inconvenientes en estructuras anatómicas asociadas con sintomatología referida craneofacial que ha probado tener una relación causa-efecto: el tono muscular aumentado o disminuido puede producir obstrucción de la tuba auditiva en dimensiones verticales disminuidas lo que se revierte en síntomas óticos (Ramirez et al., 2008). Sin olvidar que los pacientes con pérdida de DV pueden acusar también efectos locales como algias en la ATM y en la región suprahiodea, además de efectos mucosos orales y periorales (queilitis angular), resentimiento muscular por inestabilidad, dificultad en la masticación, fonación, deglución y respiración, máxime los cambios evidentes en su estética.

La DV perdida o disminuida en aquellos pacientes que permanecen edentados por mucho tiempo y sin reposición protésica, produce un efecto de adaptación oclusiva vertical negativa en forma paulatina y sistemática; pérdida silenciosa que la neurofisiología del sistema estomatognático apropia y acepta de forma tolerante con la adaptación de las estructuras involucradas. Esta situación no puede pasar inadvertida en el momento de restituir progresivamente la DV. Cuando ésta se restaura bruscamente y sin incrementos escalonados progresivos, puede ocurrir una situación clínica asociada como consecuencia de un trayecto de cierre oclusivo final de sobrepaso y relacionada a recorridos imaginarios más largos, encontrando un tope oclusal mucho antes de lo habitual que representa un micro-trauma para la ATM como para los rebordes alveolares por el choque provocado de ambas prótesis, lo que se puede traducir en dolor estomatognático local que crónicamente genera sintomatología craneofacial y cervical difusa y referida.

\section{Síntomas óticos y cráneo-cervicales referidos asociados}

a la DV. La reconstrucción de la DV entre mandíbula y maxilar con prótesis totales puede significar el alivio de síntomas referidos en cabeza y cuello. Síntomas como ruidos subjetivos (tinitus), dolor en los oídos, mareos, disminución de la audición, oídos tapados y dolores de cabeza y cuello entre otros, pueden ser generados por una prótesis total que transgrede esta relación de altura entre la mandíbula y las maxilar. La presencia de esta sintomatología, especialmente el vértigo, en el adulto mayor puede estar correlacionado con el grado de accidentalidad. Los referentes históricos ayudan a comprender que este inconveniente con la DV además de ser un problema actual, también lo fue desde comienzos del siglo pasado. Monson y Wright en 1920 relacionaron la posición de la mandíbula y la ATM con la hipoacusia en población adulta e infantil. Goodfriend en 1933 relacionó los síntomas otológicos con la ATM; y Costen en 1934 asoció la sintomatología auricular y cráneo-sinusal con los desórdenes articulares (Síndrome de Costen) (Costen, 1934), y fue el primero en describir síntomas óticos en pacientes edentados parciales o totales y la relación de la DV con esta sintomatología. Recientemente, Bjorne et al. (1998), explican que el daño en la audición y la pérdida auditiva han sido frecuentemente asociados con la relación mandíbula-maxilar y que los síntomas de tensión como la cefalea, la tensión muscular pericraneal y cervical, como los desórdenes de sueño están fuertemente relacionados con este. 
La disfunción neuromuscular de los músculos masticatorios puede iniciar alteraciones en el sistema auditivo basados en la asociación nerviosa entre estos y los músculos del oído medio y músculos tubáricos (tensores del tímpano y del velo del paladar) por la inervación común de la rama mandibular del nervio trigémino. En tal sentido, la interacción neuromuscular compleja entre los músculos de la masticación y el oído fue denominada "Síndrome Otognático" por Myrhaug en 1964 y posteriormente "Síndrome Otomandibular" por Bernstein en 1969 y por Arlen en 1977 (Ramirez et al.). Las personas con síndrome otomandibular presentan uno o más síntomas óticos, sin patología localizada en oído, nariz o garganta, pero con uno o más músculos de la masticación en estado de constante espasmo.

En concreto, la relación de cercanía entre los músculos pterigoideo medial y el músculo tensor del velo del paladar puede ser un inconveniente cuando la DV de un paciente edentado se encuentra alterada. Este contacto no funcional entre los músculos interfiere anatómicamente con la función normal de la tuba auditiva y genera síntomas óticos. La tuba auditiva se mantiene normalmente cerrada por la presión colapsante de las masas externas compuestas de estructuras como el músculo pterigoideo medial de manera lateral, inferiormente por el músculo elevador del velo palatino e internamente por un paquete adiposo. Teniendo esto en mente, en condiciones normales, la contracción del mús- culo tensor del velo del paladar abre el lumen de la tuba mientras presiona lateralmente al músculo pterigoideo medial. Sin embargo, si el músculo pterigoideo medial se encuentra espástico o hipotónico y recogido (en DV disminuida), interrumpe la contracción del músculo tensor del velo palatino dificultando la apertura de la tuba auditiva con sus consecuentes síntomas óticos referidos (Ramirez et al.) (Fig. 3).

Por otro lado, una relación alterada de la DV entre mandíbula y maxilar tiene efectos inmediatos en la posición antero-posterior del macizo craneal y la columna cervical. No es infrecuente encontrar en personas edentadas con prótesis que no restauran la DV, quejas asociadas a cefaleas tensionales suboccipitales que no ceden ni al tratamiento farmacológico convencional ni a la terapia local con infiltraciones. Al examen intraoral se descubren prótesis totales que no restauran la DV original. La angulación anteroposterior de la articulación cráneo-cervical (en la articulación atlanto-occipital) se refleja en la posición de ventroextensión y dorsi-flexion de esta unión que depende de una manera sorprendente de la DV mandíbula y maxilar. En tal sentido, una hiperflexión posterior tiene repercusiones sobre el paquete vasculo-nervioso y estructuras musculares cervicales que irritadas producen cefaleas suboccipitales con patrones de dolor proyectado expresados justamente en los territorios dermatómicos inervados por los nervios irritados mecánicamente (nervio occipital mayor).

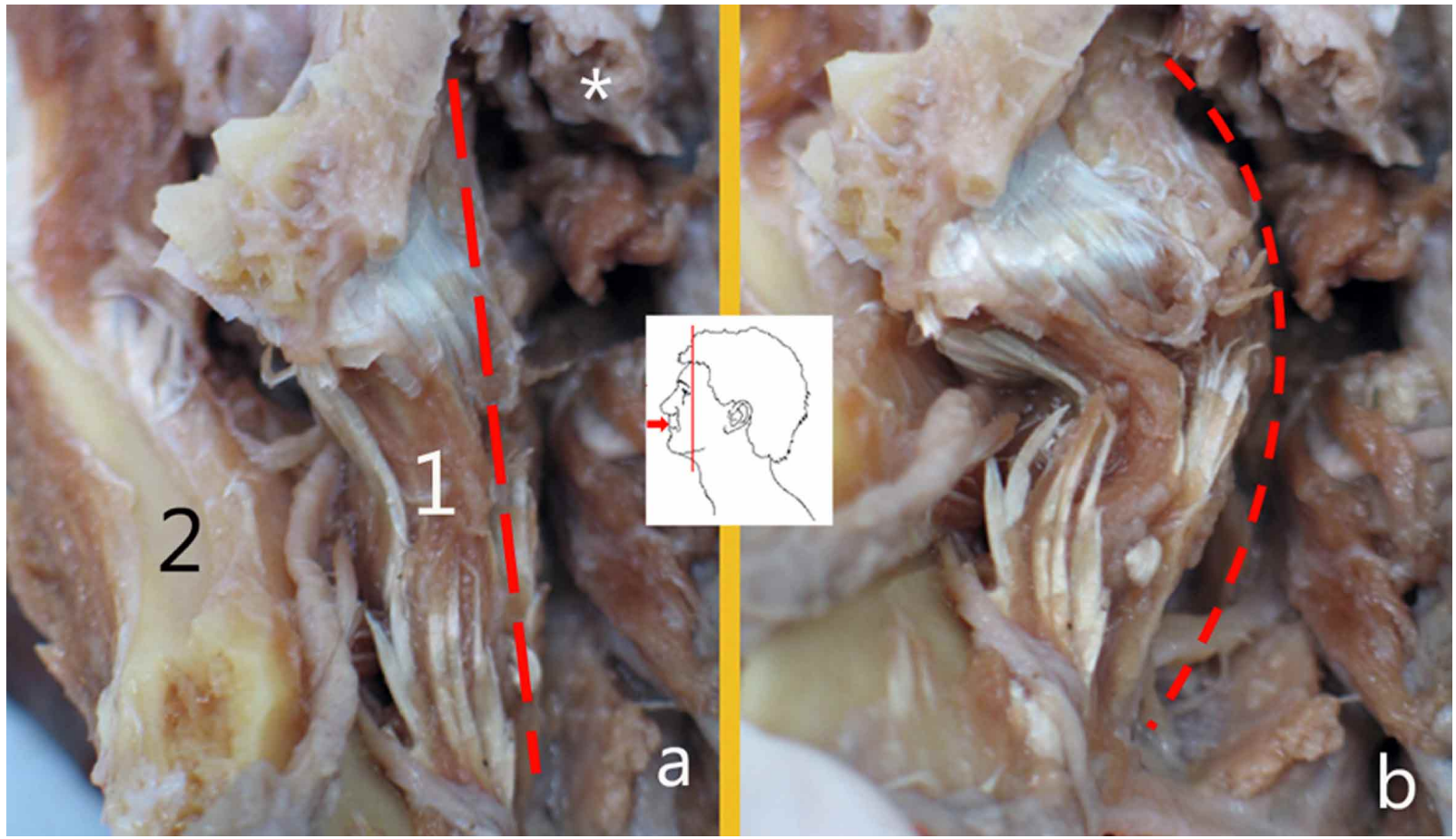

Fig. 3. Vista frontal de área faríngea y mandibular medial lado derecho: Disección del musculo pterigoideo medial y tuba auditiva. El recuadro de la mitad muestra la forma del corte. Imagen a- Mandíbula en relación vertical normal: 1. Músculo pterigoideo medial derecho, 2. Cuerpo mandibular derecho seccionado, *. Tuba auditiva. Imagen b- Mandíbula en relación vertical de sobremordida profunda incrementando el volumen transversal del músculo pterigoideo medial impactando la tuba auditiva. 
Técnicas convencionales de rehabilitación de la DV en edentados. No existe un método universalmente aceptado y disponible para medir la DV en la clínica, sin embargo, sí existe una serie de técnicas que individual y colectivamente pueden ser de utilidad en la obtención y corroboración de esta medida algo escurridiza.

A través del tiempo se ha venido evidenciando una serie de técnicas usadas por diferentes escuelas en la rehabilitación con prótesis total, que buscan una forma fiable y reproducible del registro de la DV. Algunas de ellas son medidas mecánicas o morfométricas y otras medidas fisiológicas que individualmente no son completamente íntegras y deben ser miradas con un enfoque más grupal y en el marco de una sana duda que permita corroborar algunas técnicas frente a otras avalando el registro final y aproximado de la DV. Algunas de las técnicas fisiológicas más conocidas que buscan la obtención de una posición natural de reposo (Shanahan, 2004) aproximado son: la fonética (espacio de fonación aproximado) (Zhou et al., 2010; de Souza et al., 2004), la presión máxima (origen e inserción muscular en separación optima), el umbral de deglución (de Souza et al.; Millet et al., 2003), la técnica de sensación táctil propioceptiva (con sobrepaso de la DV) (Wojdyla \& Wiederhold 2005; Massad et al., 2004; Medwedeff, 1983), y la cantidad de dientes que se muestra al sonreír (línea de sonrisa y sellado labial). En este grupo se ha querido usar el estimulo eléctrico muscular periférico (TENS) con resultados sobredimensionados y polémicos.

Otras técnicas entrarían en la clasificación de mecánicas y morfométricas; algunas de ellas serían: la posición dental posterior natural (Yanikoglu et al., 2005), la cefalometría cráneo-cervical (Babiuc et al., 2009; Ciftçi et al., 2005), la relación hioidea (Sierpinska et al., 2009), el uso de dispositivos mecánicos calibradores verticales (Willis, Sorensson, Dakometro, Boley) (Pitigoi-Aron et al., 2008), la posición dental, volumen tisular y el tono tisular (Boucher), la distancia a la papila incisiva de los incisivos superiores, la distancia del frenillo inferior a los incisivos inferiores (Bissasu, 1999), la distancia entre rebordes anteriores, la medición de la prótesis en uso (Bissasu; Boulos, 2007) registros previos a las extracciones, el paralelismo de los rebordes y la proporción de tercios faciales en los que se incorporan una serie de medidas de distancias sugeridas por Leonardo da Vinci: pupila a comisura labial, pupila a pupila, la altura de la nariz, tragus a cantus lateral del ojo, el doble de la medida intercantar interna de los ojos, el doble de la distancia intercantar de un ojo, cantus lateral de un ojo al cantus medial del otro ojo, la altura del pabellón auricular, la medida del extremo proximal de ultima falange del hálux hasta el extremo del índice, la altura de la palma de la mano del paciente superpuesta en el tercio inferior de la cara, la medida curvada de los labios y en mujeres la medida entre la ceja al origen frontal del escalpo.

Placa "Costen" como mecanismo de apoyo en la obtención de una DV ideal. Una forma de integrar las técnicas fisiológicas y las mecánicas es con la modificación vertical in vivo de la prótesis del paciente que permita un diagnóstico apropiado de la pérdida de la DV. Este proceso ya se ha hecho de manera directa sobre los dientes protésicos y con un material resinoso que altera irreversiblemente las prótesis que trae el paciente (Mays, 2003; Brunello \& Mandikos, 1998; Brosnihan, 2001). Esto permite hacer un registro fidedigno y sistemático en el tiempo aprovechando que el paciente usa sus prótesis modificadas con una DV calibrada entre citas. Al mismo tiempo, permite hacer modificaciones por adición o sustracción directa en las prótesis que admiten una mejor calibración de los parámetros fisiológicos (longitud de la fibra muscular) e igualmente los mecánicos (rendimiento masticatorio). Esta técnica facilita concretar medidas reales e individuales de la compleja dinámica fisiológica de cada paciente en un marco integral, aproximado, gradual y verificable para la rehabilitación del la DV en edentados. La desventaja se centra en el deterioro de las prótesis actuales sumado a la imposibilidad de su remoción de la boca si llegase a molestar en el proceso de calibración de la DV por sobrepaso.

El uso de esta técnica con una placa removible (Fig. 4), que personalmente denominamos "Costen" como distinción al visionario otorrinolaringólogo que reportó por primera vez daños colaterales estomatognáticos referidos en el paciente edentado con alteración de la DV, permite lograr igualmente las ventajas de la modificación vertical sugerida con resina directa en la prótesis, pero sin los inconvenientes asociados a esta técnica ya que permite que el paciente pueda quitársela si genera dolor o malestar hasta un nuevo control con su operador. Con esta placa oclusal removible de manufactura sencilla, se permite integrar la mayoría de las técnicas individuales y facilita un ejercicio de ensayo-error sobre el paciente sin involucrar la dimensión ética del ejercicio in vivo. La placa Costen permite una adaptación progresiva de la DV, pudiendo ser modificada en varios controles (marcas en grafito), de cada incremento progresivo (Fig. 5), y según la necesidades individuales expresadas por el paciente que permiten configurarla progresivamente con relación al referente anterior hecho en grafito, apoyándose en un el axioma fisiológico "La forma es determinada por la función".

Reflexiones finales. El registro de la DV contempla varios condicionantes a cumplir durante su procedimiento. El paciente debe estar estable y sin sintomatología local o referida tanto en mucosas como en el sistema músculo-esqueléti- 


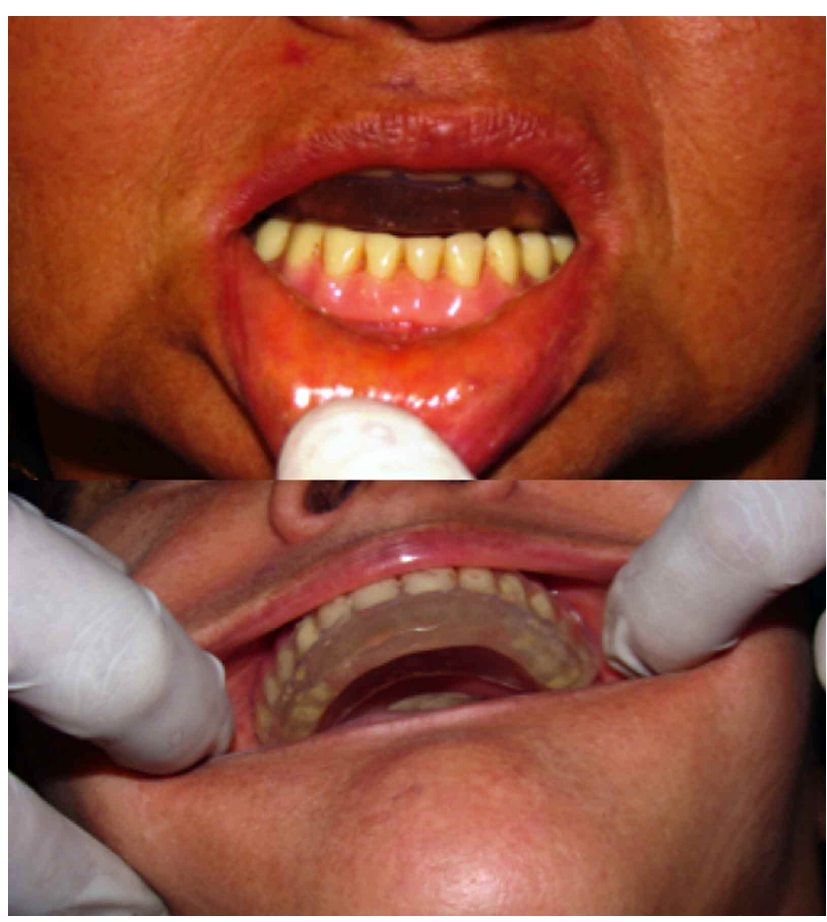

Fig. 4. Placa Costen con una DV aproximada que suple la deficiencia de altura del tercio inferior del rostro de la paciente. Fotos de consulta privada y permitidas por la paciente.

co. El dolor o la fatiga muscular modifican su registro. Adicionalmente el registro debe hacerse con el paciente tranquilo emocionalmente y sentado verticalmente en la silla. El operador debe en lo posible evitar atajos para lo que debe contar con tiempo y contemplar el error, sin avanzar y rectificando en cada control usando cuantos sean necesarios hasta lograr una relación de bienestar estable.

La prótesis total además de incluir dientes en ellas, debe brindar soporte y volumen a las mejillas y labios, contornos y dimensiones faciales (menos plegadas y arrugadas). Tiene que haber un salto de calidad en el ideal y la actitud del odontólogo que ayude a superar los paradigmas erróneos del imaginario en esta disciplina y que algunos pacientes han venido apropiando como "normales" $\sin$ serlos. Una de estas creencias clásicas es la que se necesita acostumbrar los tejidos a las prótesis nuevas ("amansarlas"), y sin contemplar la intervención activa y frecuente del operador en el proceso. Otra particular es el de no quitarse las prótesis para dormir (por razones muy personales), lo que evita la remodelación sustractiva agregada de los rebordes maxilares y permite reposar los músculos, estructuras óseas y mucosas, permitiendo un soporte biológico sin rápidas remodelaciones y mas longevo. Se debe tener en cuenta que la actividad muscular disfuncional masticatoria nocturna (bruxismo), al contrario de lo que se cree, también

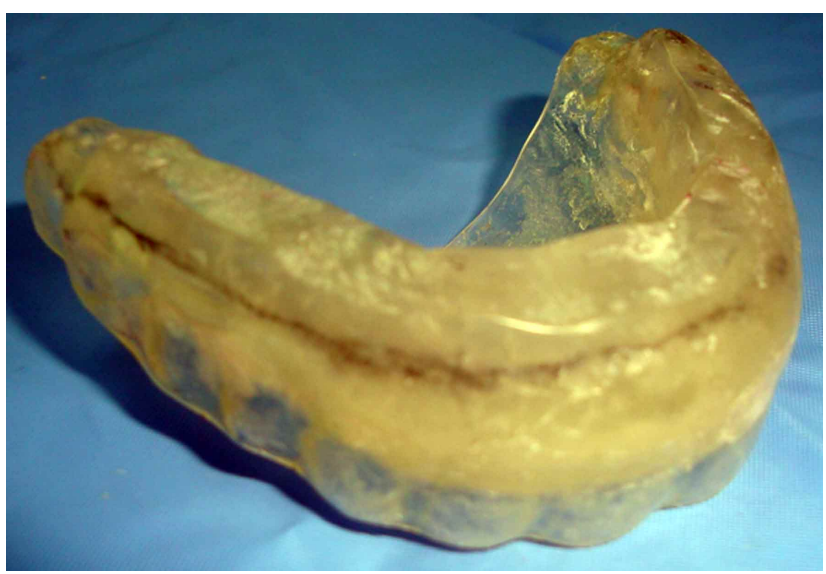

Fig. 5. Marca tangencial en Placa Costen, hecha con grafito de lápiz y previo al incremento de $2 \mathrm{~mm}$ con acrilico en la segunda cita (una semana posterior). Marca que permite un control visual de cada capa incrementada, lo que facilita la modificación del ultimo incremento conforme al reporte de malestar o bienestar del paciente.

sucede en usuarios con prótesis total y explica la sensación de apretamiento o entumecimiento de los músculos de la cara al despertar y el dolor craneofacial (desorden témporomandibular) e irritación sobre los rebordes alveolares que sufren un desgaste persistente a través del tiempo (Yannikakis et al., 2009). Finalmente, hay que considerar que la prótesis con DV errónea genera dificultad en la maceración de los alimentos con el consecuente trastorno en el funcionamiento del tracto alimenticio (funciones gastrointestinales), sin olvidar los efectos nutricionales. La dentadura total debe ser supervisada por un operador experimentado debido a la complejidad de su consolidación, calibración y mantenimiento.

La rehabilitación con prótesis total más que reclamar saberes individuales de ciencia o arte (conceptos de conocimiento), es la suma de estos y debe ser practicada con técnica, tiempo y entereza. Paralelamente y en tal sentido, la odontología como ciencia biomédica, actualmente se encuentra acoplando los rápidos cambios de los dominios tecnológicos y biólogos que seguramente significarán asimismo un cambio en la mentalidad de sus practicantes. Hay una imperante necesidad de entender las relaciones interdentales desde una perspectiva general cráneo-cervical y sistémica (estomatología) y no exclusivamente dental (odontología) y la prótesis total hace parte de este panorama actual. Las ciencias básicas serán los actores que orienten al futuro clínico en una percepción fresca y en la que la perspectiva molecular, neurofisiológica, fisiopatológica y musculo-esquelética sean los principales intérpretes de la conceptualización de un sistema estomatognático altamente complejo e intrincadamente articulado al resto del cuerpo. 
RAMÍREZ, L. M.; ECHEVERRÍA, P.; ZEA, F. J. \& BALLESTEROS, L. E. Vertical dimension on edentulous patient: relationship with symptoms reported. Int. J. Morphol., 31(2):672-680, 2013.

SUMMARY: Vertical dimension is an approximate measure of the physiological inter-maxillary relations. Its register is a sensitive and methodical process with craniofacial side effects when wrongly achieved. Single concepts for vertical dimension register should be changed and understood in a collective manner. The complexity of the masticatory dynamics requires a broader view of this notion. The correspondence between the vertical dimension and the functional-dysfunctional cranio-cervical relations is undeniable and goes beyond an exclusively dental relation. This review seeks to place the reader in a stomatognathic functional perspective, which requires greater efforts of the prosthodontics operator. Normal functioning of the stomatognathic system and surrounding structures in the edentulous patient depends on the conceptualization about the importance of vertical inter-maxillary relations

KEY WORDS: Vertical dimension; Referred symptoms; Otic symptoms; Headache; Splint; Miotatic mechanisms; Craneocervical relations.

\section{REFERENCIAS BIBLIOGRÁFICAS}

Babiuc, I.; Pa una, M.; Malit,a, M. A.; Ariton, S. G.; Damian, M.; Ungureanu, V. \& Petrini, A. Correct complete denture rehabilitation, a chance for recovering abused tissues. Rom. $J$. Morphol. Embryol., 50(4):707-12, 2009.

Bissasu, M. Use of lingual frenum in determining the original vertical position of mandibular anterior teeth. J. Prosthet. Dent., 82(2):177-81, 1999.

Bjorne, A.; Berven, A. \& Agerberg, G. Cervical signs and symptoms in patients with Meniere's disease: a controlled study. Cranio, 16(3):194-202, 1998.

Boucher, C. O. Complete denture prosthodontics--the state of the art. J. Prosthet. Dent., 92(4):309-15, 2004.

Boulos, P. J. Simplified method for recording maxillomandibular relations in complete dentures. $N$. Y. State Dent. J., 73(3):24-7, 2007.

Brosnihan, J. Aiding denture construction: a new twist to an old trick. J. Am. Dent. Assoc., 132(7):1019-20, 2001.

Brunello, D. L. \& Mandikos, M. N. Construction faults, age, gender, and relative medical health: factors associated with complaints in complete denture patients. J. Prosthet. Dent., 79(5):545-54, 1998.

Costen, J. B. A syndrome of ear and sinus symptoms dependent upon disturbed function of the temporomandibular joint. 1934. Ann. Otol. Rhinol. Laryngol., 106(10 Pt. 1):805-19, 1997.

Ciftçi, Y.; Kocadereli, I.; Canay, S. \& Senyilmaz, P. Cephalometric evaluation of maxillomandibular relationships in patients wearing complete dentures: a pilot study. Angle Orthod., 75(5):821-5, 2005.

de Souza, R. F.; Marra, J.; Pero, A. C. \& Compagnoni, M. A. Effect of denture fabrication and wear on closest speaking space and interocclusal distance during deglutition. J. Prosthet. Dent., 97(6):381-8, 2007.
Makofsky, H. W. A multidisciplinary approach to the evaluation and treatment of temporomandibular joint and cervical spine dysfunction. Cranio, 7(3):205-13, 1989.

Manns, A.; Miralles, R. \& Palazzi, C. EMG, bite force, and elongation of the masseter muscle under isometric voluntary contractions and variations of vertical dimension. J. Prosthet. Dent., 42(6):674-82, 1979.

Massad, J. J.; Connelly, M. E.; Rudd, K. D. \& Cagna, D. R. Occlusal device for diagnostic evaluation of maxillomandibular relationships in edentulous patients: a clinical technique. $J$. Prosthet. Dent., 91(6):586-90, 2004.

Mays, K. A. Reestablishing occlusal vertical dimension using a diagnostic treatment prosthesis in the edentulous patient: a clinical report. J. Prosthodont., 12(1):30-6, 2003.

Medwedeff, F. M. Oral reconstruction: an instrument to establish vertical and physiologic occlusion. J. Am. Dent. Assoc., 107(3):443-5, 1983.

Millet, C.; Jeannin, C.; Vincent, B. \& Malquarti, G. Report on the determination of occlusal vertical dimension and centric relation using swallowing in edentulous patients. J. Oral Rehabil., 30(11):1118-22, 2003.

Pitigoi-Aron, G.; Labarre, E.; Giusti, L. \& Leknius, C. Fabrication of a caliper device for interalveolar dimension measurement in the complete denture patient. J. Prosthodont., 17(6):508-10, 2008 .

Ramirez, L. M.; Ballesteros, L. E. \& Sandoval, G. P. Topical review: temporomandibular disorders in an integral otic symptom model. Int. J. Audiol., 47(4):215-27, 2008.

Shanahan, T. E. Physiologic vertical dimension and centric relation. J. Prosthet. Dent., 91(3):206-9, 2004.

Sierpinska, T.; Golebiewska, M.; Kuc, J. \& Lapuc, M. The influence 
RAMíREZ, L. M.; ECHEVERRíA, P.; ZEA, F. J. \& BALLESTEROS, L. E. Dimensión vertical en edentados: relación con síntomas referidos. Int. J. Morphol., 31(2):672-680, 2013.

of the occlusal vertical dimension on masticatory muscle activities and hyoid bone position in complete denture wearers. Adv. Med. Sci., 54(1):104-8, 2009.

Wojdyla, S. M. \& Wiederhold, D. M. Using intraoral Gothic arch tracing to balance full dentures and determine centric relation and occlusal vertical dimension. Dent. Today, 24(12):74-7, 2005.

Yanikoglu, N. D.; Güldag, M. U. \& Duymus, Z. Y. Determination of the occlusal vertical dimension: use of maxillary and mandibular posterior teeth measurement in edentate subjects. Eur. J. Prosthodont. Restor. Dent., 13(2):75-7, 2005.

Yannikakis, S.; Zissis, A.; Harrison, A. The prevalence of temporomandibular disorders among two ---different denturewearing populations. Eur. J. Prosthodont. Restor. Dent., 17:3540, 2009

Yoshino, G.; Higashi, K. \& Nakamura, T. Changes in weight distribution at the feet due to occlusal supporting zone loss during clenching. Cranio, 21(4):271-8, 2003.

Zhou, C.; Matsukawa, T.; Shimokawara, S.; Sone, M.; Yamamoto, H.; Ohmori, K.; et al. Influence of experimental palatal plate on mandibular position during continuous [n] phonation and at the physiologic rest position. Odontology, 98(2):139-43, 2010 .
Dirección para Correspondencia:

Luis Miguel Ramirez Aristeguieta, DDs, Ms, MSc.

Profesor Universidad de Antioquia

Calle 70 No. 52-21

Medellin

COLOMBIA

Tel: $57+315-7523178$

Email: Imra3@yahoo.com

Recibido : 29-08-2011

Aceptado: 08-08-2012 